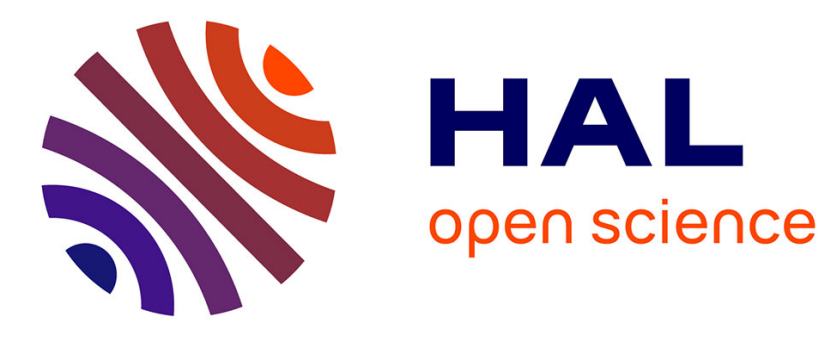

\title{
Wearable impedance monitoring system for dialysis patients
}

Stephane Bonnet, A. Bourgerette, S. Gharbi, C. Rubeck, W. Arkouche, B. Massot, Eric Mcadams, A. Montalibet, P. Jallon

\section{- To cite this version:}

Stephane Bonnet, A. Bourgerette, S. Gharbi, C. Rubeck, W. Arkouche, et al.. Wearable impedance monitoring system for dialysis patients. 2016 38th Annual International Conference of the IEEE Engineering in Medicine and Biology Society (EMBC), Aug 2016, Orlando, United States. 10.1109/EMBC.2016.7591898 . hal-01985483

\section{HAL Id: hal-01985483 https://hal.science/hal-01985483}

Submitted on 12 Apr 2019

HAL is a multi-disciplinary open access archive for the deposit and dissemination of scientific research documents, whether they are published or not. The documents may come from teaching and research institutions in France or abroad, or from public or private research centers.
L'archive ouverte pluridisciplinaire $\mathbf{H A L}$, est destinée au dépôt et à la diffusion de documents scientifiques de niveau recherche, publiés ou non, émanant des établissements d'enseignement et de recherche français ou étrangers, des laboratoires publics ou privés. 


\title{
Wearable Impedance Monitoring system for dialysis patients
}

\author{
S. Bonnet, A. Bourgerette, S. Gharbi, C. Rubeck, W. Arkouche, B. Massot, \\ E. McAdams, A. Montalibet, P. Jallon
}

\begin{abstract}
This paper describes the development and the validation of a prototype wearable miniaturized impedance monitoring system for remote monitoring in home-based dialysis patients. This device is intended to assess the hydration status of dialysis patients using calf impedance measurements. The system is based on the low-power AD8302 component. The impedance calibration procedure is described together with the Cole parameter estimation and the hydric volume estimation. Results are given on a test cell to validate the design and on preliminary calf measurements showing Cole parameter variations during hemodialysis.
\end{abstract}

\section{INTRODUCTION}

In conventional body composition studies, a fourelectrode measurement technique is generally used. In whole-body impedance analysis (WBIA), the currentinjecting electrodes are placed on the right side, with one electrode at the right hand, and the other electrode at the foot [1]. A pair of voltage-measuring electrodes is placed within the current-injecting electrodes. The four-electrode technique works on the principle that the two outer electrodes drive a relatively homogeneously distributed current through the tissue under study. Two inner electrodes are used to measure the voltage drop across the tissue of interest, sandwiched directly between them. The advantage of this technique is that the four electrode-gel-skin interfaces do not influence the measurement of the targeted 'core' tissue impedance.

An alternative is to consider segmental BIA with electrodes placed on a single limb. The calf location seems well suited for monitoring hydration [2]. Electrodes could easily be placed into a garment and the body composition could be monitored during long period of time. In order to envisage such long-term monitoring, a wearable miniaturized device has to be developed. Specifications are mostly in terms of power consumption, weight and ergonomics. Moreover, the system must be able to perform bioimpedance spectroscopy measurements. Multifrequency BIA allows estimating the extracellular and intracellular fluid (ECF/ICF) volume compartment.

\footnotetext{
*Research supported by ANR Dialydom project and FP7 Swan iCare European project.

S. Bonnet is with Univ. Grenoble Alpes, F-38000 Grenoble, France. CEA LETI MINATEC Campus, F-38054 Grenoble France (tel: 33 (0)4 38 7840 70; fax: 33 (0)4 387854 56; e-mail: stephane.bonnet@cea.fr).

A. Bourgerette, S. Gharbi, C. Rubeck, P. Jallon (corresponding author) are also with CEA LETI.

W. Arkouche is with AURAL,F-69008 Lyon, France

B. Massot, E. McAdams, A. Montalibet are with the Institut des Nanotechnologies de Lyon, INL-UMR5270, INSA Lyon, F-69100 Lyon, France
}

P. Bogónez -Franco [3], has found that commercially available devices are still sensitive to electrode-skin contact impedance, especially when used for localized measurements with their associated smaller tissue impedances. As the length of the skin segment measured is decreased, electrode contact impedance plays a more important role and adversely influences the accuracy of the impedances measured, causing gross distortion of the measured impedance loci - hence the importance of using multi-frequency impedance measurements to detect these distortions. It is therefore very important to achieve a contact impedance as low as possible, for example by using electrodes with larger contact areas and, of course, by improving the measurement circuitry.

This paper deals with the measurement system that has been developed for this application. It is described in Section II together with the calibration phase together with the highfrequency compensation. Section III describes the Cole parameter estimation technique that has been used and that can be embedded in the firmware. Section IV presents results obtained with this device with test cells and using calf impedance measurements.

\section{SYSTEM DESCRIPTION}

\section{A. Impedancemeter}

The low-power programmable waveform generator AD9837 is used to establish a $210 \mathrm{mV}$ sinusoidal voltage (2$500 \mathrm{kHz}$ ). This voltage is converted by a VCCS into a sinusoidal current of either $70 \mu \mathrm{A}_{\text {rms }}$ or $200 \mu \mathrm{A}_{\mathrm{rms}}$ by the transconductance amplifier OPA861. The current is applied to the stimulation electrodes (I+/I). The injected current (MesIz) and the measurement voltage at measurement electrodes $(\mathrm{Mes} V z)$ are amplified by a custom-designed amplifier circuit. The component AD8302 is finally used to extract the modulus and phase difference between the two signals for each frequency. By the trigonometric functions of the MSP430 microcontroller, the real and imaginary parts of the impedance may finally be calculated $R=|Z| \cos \varphi_{z}, X=|Z| \sin \varphi_{z}$. The impedance spectrum is stored into several memory blocks of the microcontroller and may be transferred to a terminal (e.g. PC or smartphone) via USB or Bluetooth, on demand.

The HOMEDEVICE impedance system allows the measurement of 128 frequencies in less than 10 seconds. A Low Energy Bluetooth connection is available. A smartphone application has been developed to receive the data from the impedance device. An isolated USB port is also available. The device is powered by an internal battery for safety. 


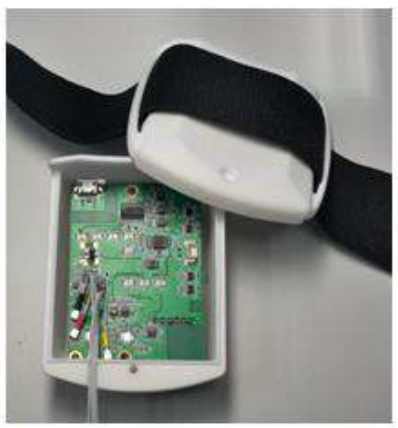

Figure 1. Electronic board of the impedancemeter (dimensions are $70 \times 50 \mathrm{~mm}$ ).

\section{B. Calibration}

The AD8302 returns the continuous voltage $V_{M A G}=$ $\alpha_{M A G} \log _{10}\left[\frac{\left\langle H_{v} v_{z}\right\rangle}{\left\langle H_{i} i_{z}\right\rangle}\right]+\beta_{M A G}$ where $\langle$.$\rangle indicates the RMS of$ the signal. The coefficients $\left(\alpha_{M A G}, \beta_{M A G}\right)$ are obtained in a preliminary calibration step using known signals.

In the proposed impedancemeter, the transfer function $H_{i}$ is automatically adapted by adjusting a register value $D_{W 3}$ so that the ratio of RMS values is approximately unity. Using the relation $\frac{\left\langle H_{v} v_{z}\right\rangle}{\left\langle H_{i} i_{z}\right\rangle}=\left|\frac{H_{v}}{H_{i}}\right||Z|$, the calibration consists in determining the regression coefficients $\left(\alpha_{Z}, \beta_{Z}\right)$ such that $\left|\frac{H_{i}}{H_{v}}\right|=\alpha_{Z} D_{W 3}+\beta_{Z}$. The impedance module is finally estimated as

$$
|Z|=10^{\frac{\alpha_{M A G}}{V_{M A G}-\beta_{M A G}}}\left(\alpha_{Z} D_{W 3}+\beta_{Z}\right)
$$

\section{Phase compensation}

Artefacts are seen on phase measurements at high frequency with the so-called hook effect [4]. Some authors have proposed to correct the complex impedance measurements by adding a frequency-dependent phase term: $\hat{Z}(\omega)=Z_{\text {meas }}(\omega) e^{-j \omega T_{d}}$. The $T_{d}$ term is considered to be a simple time delay. The parameters of the expanded Cole model are obtained using a nonlinear complex data fit. In our case, we have implemented the approach of Buendia $\hat{Z}(\omega)=\frac{Z_{\text {meas }}(\omega)}{1-j Z_{\text {meas }}(\omega) \hat{C}_{\text {par }} \omega}$ where the parasitic capacitance $C_{p a r}$ is easily estimated by regression from the susceptance spectrum.

\section{METHODS}

A typical Cole-Cole plot obtained with our impedance system (frequency range: $2 \mathrm{kHz}-500 \mathrm{kHz}$ ) is shown in Fig. 2.

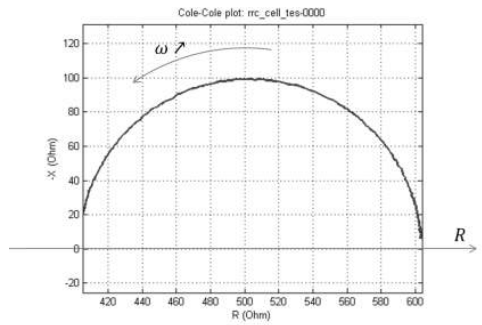

Figure 2. Cole-Cole plot on a $\mathrm{R} / /(\mathrm{R}+\mathrm{C})$ cell test.
As it can be observed, the Cole-Cole plot is distributed along a circular arc. Each point in the impedance plane ( $\mathrm{x}$ is the resistance part, $\mathrm{y}$ is the (negative) reactance part) corresponds to a measurement at a given frequency. Highfrequency points are on the left hand side, close to the origin.

\section{A. Circular fit}

The Kasa method is used to fit a semi-circle to the impedance measurements. The objective criterion is given by $\phi\left(x_{c}, y_{c}, r\right)=\sum_{n=1}^{N} \phi_{n}^{2} \quad$ where $\quad \phi_{n}=\left\|\mathbf{x}_{n}-\mathbf{c}\right\|^{2}-r^{2}$ denotes the algebraic distance of the point $\mathbf{x}_{n}$ to the circle. The partial derivatives of the cost function are nonlinear with respect to the input variables. By making the change of variables, $u=-2 x_{c}, v=-2 y_{c}, w=x_{c}^{2}+y_{c}^{2}-r^{2}$ we can rewrite the cost function as $\phi(u, v, w)=\sum_{n=1}^{N}\left[z_{n}+u x_{n}+\right.$ $\left.v y_{n}+w\right]^{2}$ where $z_{n}=x_{n}^{2}+y_{n}^{2}$. Setting the partial derivatives to zero, yields a $3 \times 3$ linear system of equations, for 3 unknowns:

$$
\left(\begin{array}{ccc}
\overline{x x} & \overline{x y} & \bar{x} \\
\overline{x y} & \overline{y y} & \bar{y} \\
\bar{x} & \bar{y} & 1
\end{array}\right)\left(\begin{array}{c}
u \\
v \\
w
\end{array}\right)=\left(\begin{array}{c}
-\overline{x z} \\
-\overline{y z} \\
-\bar{z}
\end{array}\right)
$$

where $\bar{x}$ means the average value of the quantity $x$.

The variables of interest are finally recovered by computing $x_{c}=-\frac{u}{2}, y_{c}=-\frac{v}{2}, r=\frac{\sqrt{u^{2}+v^{2}-4 w}}{2}$

\section{B. Impedance parameter estimation}

Once a circle has been fitted to the impedance measurements, it is then possible to extract relevant parameters. The Cole model is given by

$$
Z=R_{\infty}+\frac{R_{0}-R_{\infty}}{1+(j \tau \omega)^{\alpha}}
$$

By denoting $u=Z-R_{\infty}, v=(j \tau \omega)^{\alpha} u$, we obtain $u+v=$ $R_{0}-R_{\infty}$ and $\arg v-\arg u=\alpha \frac{\pi}{2}$. According to the inscribed angle theorem, the set of points $M(Z)$ in the complex plane for which the angle $R_{0} M R_{\infty}$ is constant is an arc of circle. According to Fig. 3 , the angle $\measuredangle\left(R_{\infty} M R_{0}\right)=$ $\pi-\alpha \frac{\pi}{2}$ and thus the measure of the angle $\measuredangle\left(R_{\infty} O R_{0}\right)=$ $2 \pi-\alpha \pi$.

The locus of the impedance measurements is a semicircular arc where the center of the semicircle is located beneath the real axis. The characteristic pulsation is given by $\omega_{c}=1 / \tau$ and corresponds to the apex of the semi-circle.

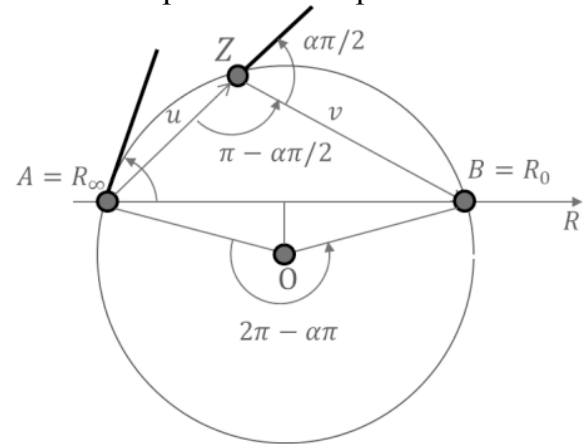

Figure 3. Cole-Cole plot on a $\mathrm{R} / /(\mathrm{R}+\mathrm{C})$ cell test. 
From these relations, we may deduce the following algorithm to compute the intersections of the real axis (impedance axis) to obtain an estimate of $R_{D C}$ and $R_{\infty}$.

The resistances are obtained using Pythagorean theorem $\left(R_{0}, R_{\infty}\right)=R_{c} \pm \sqrt{r^{2}-X_{c}}$. The $\alpha$ angle is obtained using the relation $(1-\alpha) \frac{\pi}{2}=\operatorname{atan}\left(\frac{X_{c}}{R_{c}-R_{\infty}}\right)$. Finally, the time constant $\tau$ is obtained by noting that

$$
\ln \left|\frac{v}{u}\right|=\ln \left|\frac{R_{0}-Z}{Z-R_{\infty}}\right|=\alpha \ln (\tau \omega)
$$

\section{Hydric volume estimation}

From these values, it is possible to estimate fluid volumes using the fundamental relation $R=\rho l / S$ with $l$ the length between the inner electrodes. By considering the calf as a homogeneous conductive cylinder, one obtains $V=S l=$ $\rho l^{2} / R$. In order to take into account the medium inhomogeneity (muscle, fat), an apparent resistivity is introduced. At low frequencies, it is defined as $\rho=$ $\left(\frac{V}{V_{e}}\right)^{3 / 2} \rho_{e}$ where $V$ is the calf volume. We obtain

$$
V_{e}^{3 / 2}=\frac{\rho_{e}}{R_{e}} V^{1 / 2} l^{2}
$$

where $V_{e}$ is the extracellular calf volume.

This methodology can be extended to estimate the total calf water by introducing an equivalent resistivity $\rho_{\infty}$. The ratio is thus related to the percentage of extracellular fluid.

$$
\left(\frac{V_{e}}{V_{t}}\right)^{\frac{3}{2}}=\frac{\rho_{e} R_{\infty}}{\rho_{\infty} R_{e}}
$$

where $V_{t}$ is the total calf volume, including both extracellular and intracellular volume.

The resistivity $\rho_{e}$ has been calibrated using Bromide dilution techniques and $\rho_{\infty}$ has been calibrated using dual absorptiometry (DXA) [5].

\section{TABLE I. APPARENT RESISTIVITIES}

\begin{tabular}{llll} 
& men & women & Estimation technique \\
\hline$\rho_{e}(\Omega . \mathrm{cm})$ & 40.3 & 42.3 & Bromide dilution technique \\
$\rho_{\infty}(\Omega . \mathrm{cm})$ & 104.3 & 100.5 & DXA
\end{tabular}

\section{RESULTS}

\section{A. Test cell}

The Impedimed SFB7 [6] Test Cell is a test circuit that consists of the resistance $R_{e}=604 \Omega$ (ECF) wired in parallel with the resistance $R_{i}=1211 \Omega$ (ICF), where the current must pass the capacitance $C_{m}=1 \mathrm{nF}$ (from the cell membranes) to run through $R_{i}:$ it is abbreviated as $R_{e} \|\left(R_{i}+C_{m}\right)$. At very low frequency, $R_{D C}=R_{e}$ and at very high frequency, $R_{\infty}=R_{e} R_{i} /\left(R_{e}+R_{i}\right)=403 \Omega$. The cell test is conform to the Cole model since

$Z=\frac{R_{e}\left(R_{i}+Z_{C}\right)}{R_{e}+\left(R_{i}+Z_{C}\right)}=R_{\infty}+\frac{R_{e}-R_{\infty}}{1+\left(R_{e}+R_{i}\right) Z_{C}^{-1}}$

where $Z_{C}^{-1}=j C_{m} \omega$. This gives $\tau=\left(R_{e}+R_{i}\right) C_{m}=1.8 \mu \mathrm{s}$. The Cole-Cole plot is given at Fig. 4

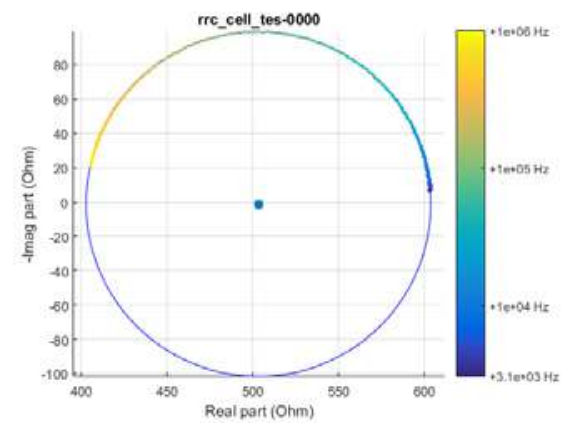

Figure 4. Circular fit for cell test.

Raw impedance points (color), fitted circle (blue).

The extracted parameters using the Kasa method are $\left(x_{c}, y_{c}\right)=(503.1 \Omega,-1.26 \Omega)$ and $r=100.6 \Omega$ from which we estimate $R_{e}=603.7 \Omega, R_{i}=1208.1 \Omega, \alpha=0.99, \tau=$ $1.7 \mu \mathrm{s}$. The agreement is quite good between the theoretical and experimental values. Note that these experimental data have been collected with the SFB7 (Impedimed) to validate the circular fit and the Cole parameter extraction algorithms (256 frequencies, $200 \mathrm{uArms}, 4-1000 \mathrm{kHz}$ ).

\section{B. Calf measurement}

The proposed method has been applied on a volunteer subject, as illustrated in Fig.5. The HOMEDEVICE (white box, strapped onto the calf) is connected to current-injecting and voltage-measurement hydrogel electrodes, developed by INSA-Lyon.

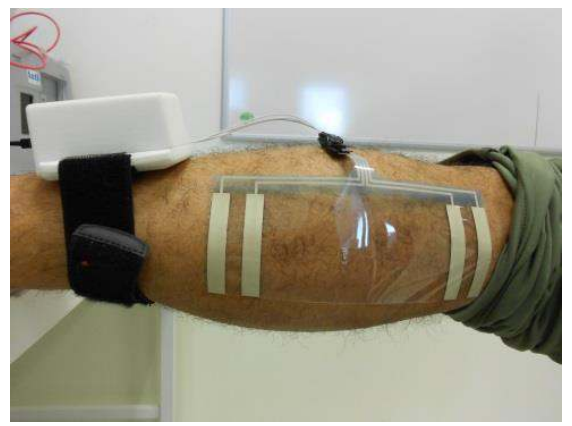

Figure 5. Calf bioimpedance measurement on a volunteer subject.

As can be seen in Fig. 6, the bioimpedance data measurements follow a circular locus.

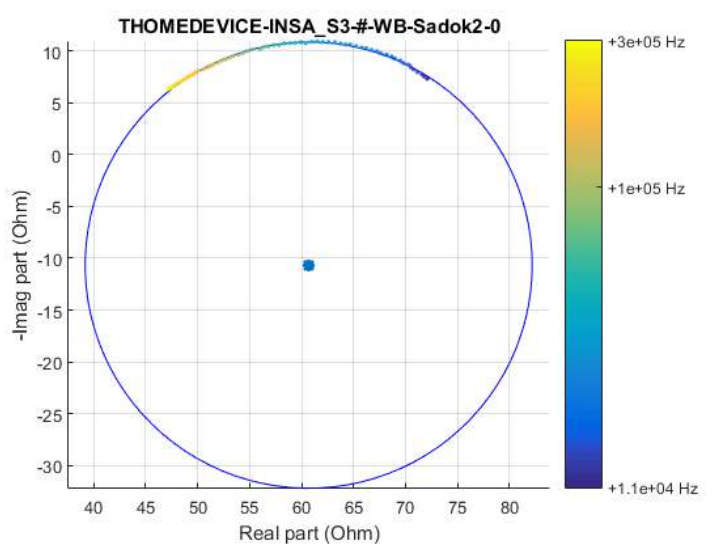

Figure 6. Circular fit for segmental impedance. Raw impedance points (color), fitted circle (blue). 
For this measurement, the extracted parameters using the Kasa method are given below: $\left(x_{c}, y_{c}\right)=(60.7 \Omega,-10.7 \Omega)$ and $r=21.5 \Omega$ from which we estimate $R_{e}=79.3 \Omega, R_{i}=$ $89.3 \Omega, \alpha=0.67, \tau=3.6 \mu \mathrm{s}$. The center is located beneath the real line and the impedance values have a $60 \Omega$ magnitude.

Note that the time constant can also be estimated from the apex determination which gives the characteristics pulsation. This method is not preferred here since it relies only on one point. Also it has been observed that the alpha angle obtained using geometry gives better fit compared to alpha deduced by regression. We plot in Fig. 7, the experimental values in the regression of (4) from which the time constant is estimated.

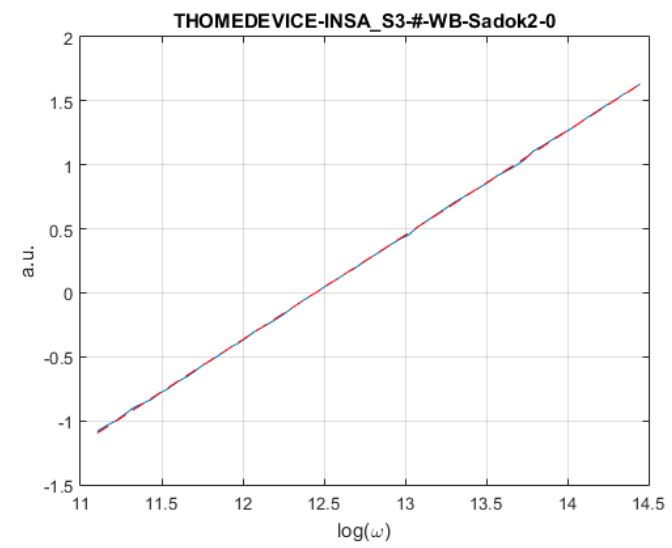

Figure 7. Linear regression plot to obtain alpha and tau.

The phase compensation is illustrated in Fig. 8 on real data from the HOMEDEVICE. It can be appreciated that the phase measurement at high frequencies are correctly rectified according to the method described by Buendia [4].

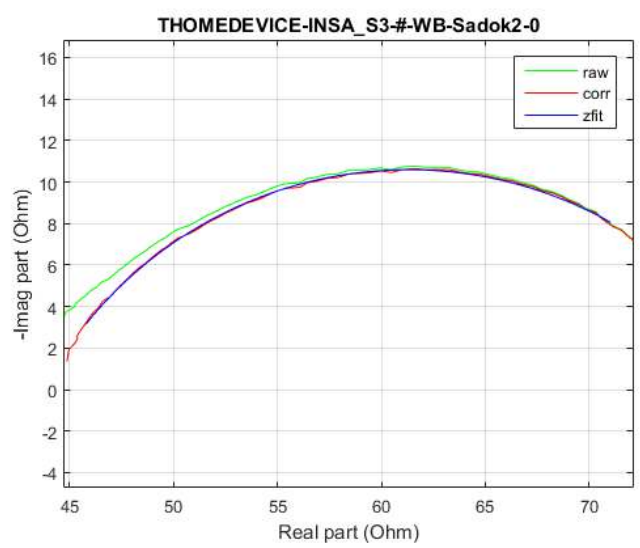

Figure 8. Raw calf impedance measurement (green), corrected (red) and fitted measurement (blue)

\section{Hemodialysis measurement}

The proposed method has been applied on a patient under hemodialysis (HD), male, age $=54 \mathrm{y}, \mathrm{BMI}=23.4$. Three WBIA impedance measurements at different time instants of the HD are considered. The Impedimed SFB7 system has been used since this device is certified for human studies. We see in Fig. 9 that both quantities $R_{e}, R_{i}$ are modified. This could be explained by the fact that fluids from both compartments are extracted during HD and this will result is an impedance increase as expected. Another explanation could be the fluid shift (after $10 \mathrm{~min}$ ) due to the person changing from vertical to horizontal posture during HD.

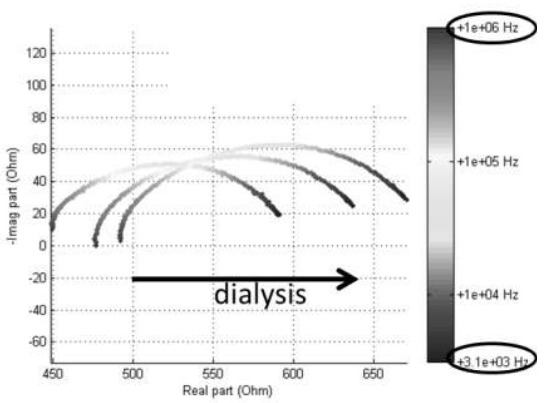

Figure 9. Whole-body bioimpedance spectra during hemodialysis.

\section{CONCLUSION}

The paper describes an integrated and wireless impedance monitoring system based on localized measurements on the calf. The first tests in our laboratory show a good agreement between theoretical and experimental values from the SFB7 (Impedimed SFB7). We plan in the future to do a comparison (or a conversion factor that enables comparison) between the estimates obtained with the HOMEDEVICE and the SFB7. The HOMEDEVICE was tested and calfbased calculations were made. When the HOMEDEVICE will be certified, we aim to collect data during long-term monitoring of patients between their dialysis.

\section{ACKNOWLEDGMENT}

The authors would like to thank the Agence Nationale de la Recherche for funding the DIALYDOM project (grant number ANR-12-TECS-0003). The authors would like to thank the Swan iCare project (FP7-317894) co-funded by the European Commission under the Information and Communication Technologies 7th Framework Program.

\section{REFERENCES}

[1] P.W. Chamney et al., "A new technique for establishing dry weight in hemodialysis patients via whole body bioimpedance," Kidney Int., vol. 61, pp. 2250-2258, 2002

[2] F. Zhu et al., "Estimation of normal hydration in dialysis patients using whole body and calf bioimpedance analysis," Physiol. Meas., vol. 32, pp. 887-902, 2011.

[3] P. Bogónez-Franco et al., "Effect of electrode contact impedance mismatch on 4-electrode measurements of small body segments using commercial BIA devices," in 18th International Workshop on ADC Modelling and Testing Research on Electric and Electronic Measurement for the Economic Upturn, Benevento, Italy, September $15-17,2014$

[4] Buendia R, Seoane F, Gil-Pita, "Experimental validation of a method for removing the capacitive leakage artifact from electrical bioimpedance spectroscopy measurements," Meas. Sci. Technol., vol. 21, 115802 (8pp), 2010

[5] Jaffrin MY, Morel H, "Body fluid volumes measurements by bioimpedance: a review of bioimpedance spectroscopy (BIS) and bioimpedance analysis (BIA) methods," Med. Eng. Phys., vol. 30, pp. 1257-1269, 2008

[6] SFB7, https://www.impedimed.com/products/sfb7-for-bodycomposition/ 